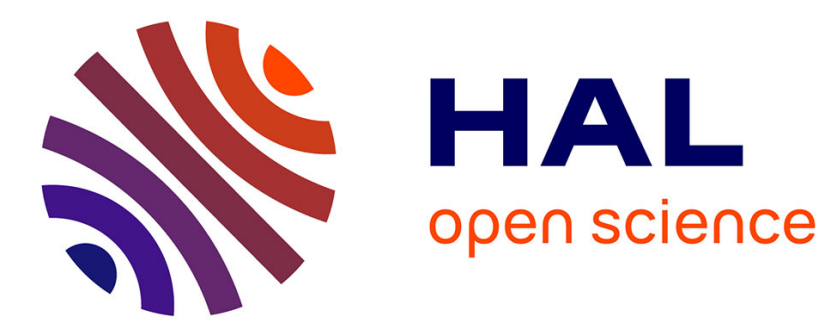

\title{
Contact and macroscopic ageing in colloidal suspensions
}

Francesco Bonacci, Xavier Chateau, Eric M Furst, Jennifer Fusier, Julie

Goyon, Anaël Lemaître

\section{To cite this version:}

Francesco Bonacci, Xavier Chateau, Eric M Furst, Jennifer Fusier, Julie Goyon, et al.. Contact and macroscopic ageing in colloidal suspensions. Nature Materials, 2020, 19 (7), pp.775-780. 10.1038/s41563-020-0624-9 . hal-02938734

\section{HAL Id: hal-02938734 \\ https://hal.science/hal-02938734}

Submitted on 15 Sep 2020

HAL is a multi-disciplinary open access archive for the deposit and dissemination of scientific research documents, whether they are published or not. The documents may come from teaching and research institutions in France or abroad, or from public or private research centers.
L'archive ouverte pluridisciplinaire HAL, est destinée au dépôt et à la diffusion de documents scientifiques de niveau recherche, publiés ou non, émanant des établissements d'enseignement et de recherche français ou étrangers, des laboratoires publics ou privés. 


\title{
Contact and macroscopic aging in colloidal suspensions
}

\author{
Francesco Bonacci, ${ }^{1}$ Xavier Chateau, ${ }^{1}$ Eric M. Furst, ${ }^{2}$ Julie Goyon, ${ }^{1}$ Jennifer Fusier, ${ }^{1}$ and Anaël Lemaître ${ }^{1}$ \\ ${ }^{1}$ NAVIER, UMR 8205, École des Ponts ParisTech, \\ IFSTTAR, CNRS, UPE, Champs-sur-Marne, France \\ ${ }^{2}$ Department of Chemical and Biomolecular Engineering, \\ University of Delaware, 150 Academy Street, Newark, Delaware, 19716, USA
}

(Dated: September 15, 2020)

\begin{abstract}
The aging behaviour of dense suspensions or pastes at rest is almost exclusively attributed to structural dynamics. Here, we identify another aging process, contactcontrolled aging, consisting in the progressive stiffening of solid-solid contacts, after their initial formation rapidly arrests the microstructure. By combining rheometry, confocal microscopy, and particle-scale mechanical tests using laser tweezers, we demonstrate that this process governs the shear modulus aging of dense aqueous silica and polymer latex suspensions at moderate ionic strengths. We further show that contact-controlled aging becomes relevant as soon as Coulombic interactions are sufficiently screened out that the formation of solid-solid contacts is not limited by activation barriers. Given that this condition only requires moderate ion concentrations, contact-controlled aging should be generic in a wide class of materials, such as cements, soils, or 3D inks, thus questioning our understanding of aging dynamics in these systems.
\end{abstract}

Dense colloidal suspensions (or pastes) constitute a broad class of materials found in areas ranging from environmental systems (e.g. silts, clays), to industry (ceramics, drilling muds, slurries), construction (plaster, cements), foodstuff, cosmetics, pharmaceuticals (toothpaste, medical ceramics). Their most remarkable feature is thixotropy: a slow evolution of mechanical properties when switching between rest and flow (at fixed density, in the absence of drainage) [1-7]. Under flow, their viscosity depends on time and strain history; at rest, their shear modulus and yield stress often display aging, i.e. slow, non-exponential long-time dynamics.

In recent decades, tremendous progress has been made towards understanding the dynamics of so-called "stabilized" suspensions, in which the formation of interparticle adhesive contacts is fully avoided by tuning interparticle interactions (esp. double-layer polarization); in such cases, only moderate levels of attraction may be introduced, e.g. via polymer depletion effects [8-12]. Confocal microscopy was instrumental to these advances, yet may only be applied to transparent, index matched, systems, in which van der Waals forces are essentially absent. Meanwhile, studies of "non-stabilized" suspensions have focused on very dilute systems (packing fractions at most a few percent) where a structural evolution (floc formation) could be imaged and thus analyzed using, e.g., light scattering [13-15].

The success of these studies has created an observational bias, as classical works on suspensions, and recent theoretical approaches, only consider structural dynamics as a possible cause of thixotopy [16-19]. But the pastes of civil and environmental engineering are dense and generally contain sufficient ion concentrations to screen Coulombic repulsion, thus allowing attractive van der Waals forces to bring particles into solid-solid contacts, which may significantly influence macroscopic properties and their evolution. Indeed, solid-solid friction, which is time-dependent [20-22], is known to affect the macroscopic response of non-colloidal granular materials; the formation of hydrate gels between grains [23, 24] was proposed to play a role in thixotropy [25]; and contact aging [26] was proposed to be at work in highly dilute $(0.02 \%)$ gels of nanometric particles in microgravity - a system quite far from real pastes. Yet, a direct relation between contact and macroscopic aging in an attractive suspension has never been established.

In fact, it remains unclear how solid-solid contacts may affect the elastic modulus of colloidal systems. By designing an optical-trap three-point bending test, Pantina and Furst [27, 28] showed that beams of PMMA and polystyrene particles present a finite flexural modulus, which entails that interparticle contacts resist sliding and rolling. The flexural modulus of polystyrene particle rods was later shown to evolve [29]. These two findings lead us to ask whether the evolution of the contact bending stiffness could be responsible for mechanical aging in pastes.

In this work, we measure by rheometry the shear modulus of dense hydrophilic silica suspensions flocculated by the addition of moderate concentrations of a divalent salt. We find that, for suspensions of particles of various sizes (from 0.5 to $1.9 \mathrm{\mu m}$ ), at volume fractions between 29 to $40 \%$, the macroscopic shear modulus $G^{\prime}$ grows quasilogarithmically at late times, whereas the microstructure is rapidly frozen. Using laser tweezers, we perform three point flexural tests on $1.9 \mathrm{\mu m}$ silica particle beams, at the same salt concentrations. We demonstrate that, in these conditions: (i) silica particles form irreversible solid-solid, roll-resistant, contacts; (ii) the contact bending rigidity grows quasi-logarithmically, like $G^{\prime}$, and with the same characteristic time scale. Experimental details are provided in Methods.

These observations lead us to attribute macroscopic 

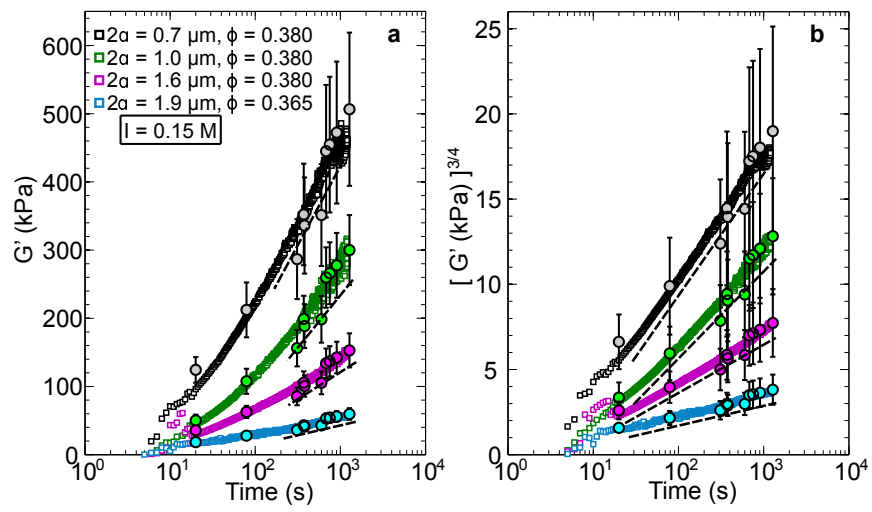

FIG. 1. Mechanical aging in dense silica suspensions. The shear modulus $G^{\prime}$ (squares) as a function of aging time, for suspensions of ionic strength $I=0.15 \mathrm{M}$, for: (i) packing fraction $\phi \simeq 38 \%$ and particle diameters, $2 a=0.7$ (black), 1 (green), and $1.6 \mu \mathrm{m}$ (purple); (ii) $\phi \simeq 36.5 \%$ and $2 a \simeq$ $1.9 \mu \mathrm{m}$ (blue). Filled circles (in lighter corresponding colors) are the predictions obtained by reconstructing (see text) $G^{\prime}(t)$ using our three-point bending tests (three rod samples). a: Log-lin plot of $G^{\prime}$ vs $t$ showing the near logarithmic growth (asymptotes in dashed lines); $\mathbf{b}:$ Log-lin plot of $\left[G^{\prime}\right]^{3 / 4}$ vs $t$ supporting the asymptotic scaling $G^{\prime} \simeq[\log (t / \tau)]^{4 / 3}$ (see text for details).

aging, in this system, to the progressive stiffening of contacts in the absence of any structural evolution. A scaling relation identified on these premices permits us to quantitatively reconstruct all of our macroscopic shear modulus data (different particle sizes) from flexural data for a single particle size. This remarkable agreement strongly supports that the growing macroscopic shear modulus is essentially determined by bending (as opposed to elongational) contact rigidity [30, 31]. Similar observations in flocculated dense suspensions of $3 \mu \mathrm{m}$ PMMA particles support the genericity of contact-driven macroscopic aging in dense flocculated suspensions.

\section{Aging without microstructural evolution}

Prior to any macroscopic aging measurement (see Methods), to insure reproducibility, our suspensions are brought into a well-defined initial state via a strong preshearing. Aging time $t$ is counted starting when the preshearing torque is set to zero. This is a slight overestimate, as a modest rotation of the inner cylinder continues for about five seconds; however, the shear modulus $G^{\prime}$ is hardly measurable until about $t \simeq 10 \mathrm{~s}$.

As seen in Fig. 1, $G^{\prime}$ increases monotonically over the accessible time range, and reaches asymptotically a quasi-logarithmic growth regime. Its magnitude decreases with the increasing particle radius $a$. Our $G^{\prime}(t)$ data also include different packing fractions $\phi$, and ionic strengths $I$ : as shown in Supplementary Note $1, G^{\prime}$ is essentially $I$-independent [7], and strongly increases with $\phi$; meanwhile, it is at least a decade larger than the loss modulus, which establishes the solid-like character of our systems.

To assess whether aging results from microstructural changes, it would be desirable to image particles, inside our suspensions, using a confocal microscope. Unfortunately, the strong index mismatch between silica $(n \approx 1.5)$ and pure water $(n \approx 1.33)$ precludes doing so beyond a few particle layers inside the system.

To gain insight on the relevance, or lack thereof, of structural evolution, we have examined suspensions of silica particles in $60 / 40 \mathrm{wt} \%$ water/glycerol mixtures. Supplementary Note 1 shows that these systems present the same quasi-logarithmic shear-modulus aging as pure water suspensions, albeit of a smaller amplitude. Fig. 2a shows a typical confocal microscopy image at depth $\approx 15 \mu \mathrm{m}$ in a water/glycerol system. Since $2 a=1.6 \mu \mathrm{m}$, this is deep enough to avoid wall effects. In Fig. 2b, we draw the particle positions reconstructed from this and later images, at three different times in the macroscopic aging regime. The disappearance of certain particles with time is an effect of the progressive bleaching of the fluorescein - particles slightly away from the focal plane become increasingly difficult to distinguish. Quite strikingly, all the particles that can be imaged at the three considered times remain essentially fixed at the same positions: the microstructure is constant during mechanical aging.

These observations establish that, in our silica suspensions in a glycerol-water mixture, shear modulus aging proceeds without structural evolution, which suggests that solid-solid contacts are rapidly formed and age. In all likelihood, the same applies to silica particle suspensions in pure water, since van der Waals forces are much larger, hence particles are more likely to form stable solid-

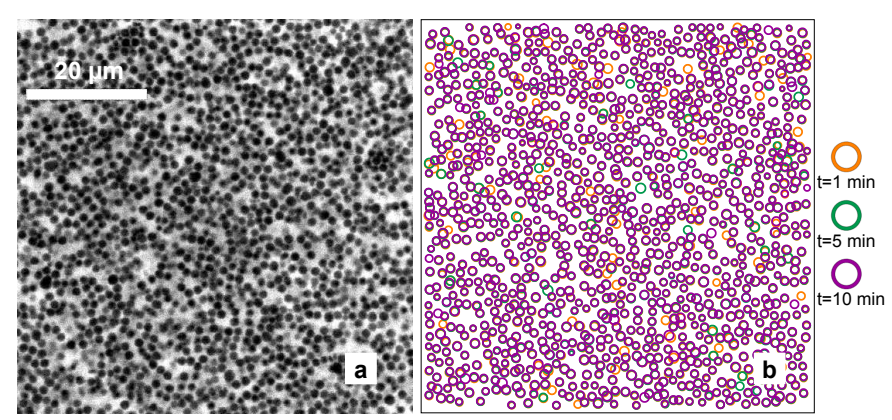

FIG. 2. Absence of structural aging in a water/glycerol mixture. a: Typical confocal image of a suspension of $2 a=1.6 \mu \mathrm{m}$ silica particles in a $60 / 40 \mathrm{wt} \%$ water/glycerol mixture, with $\phi=39 \%, I=0.15 \mathrm{M}$, in a plane parallel to the bottom cover slip, at depth $\approx 15 \mu \mathrm{m} ;$ b: Reconstructed particle positions at $t=1$ (orange), 5 (green), and 10mn (purple) after the sample was strongly pre-sheared and destructured manually with a spatula. 
solid contacts. This idea is also indirectly supported by the fact that, despite the density mismatch, sedimentation rapidly stops after cessation of pre-shearing [7]. It leads us to ask two questions:

(I) can we evidence solid-solid contact formation and aging for silica particles in pure water at relevant ionic strengths?

(II) if so, can we predict mechanical aging from contact aging, by assuming that the microstructure is rapidly fixed after arrest?

\section{Solid-solid contact stability and aging}

To address question (I), we carry out optical tweezer experiments using Pantina and Furst's method [27, 28], which consists in assembling an odd number of particles into a rod and performing three-point bending tests, as illustrated in Fig. 3a. This experiment uses particles of diameter $2 a=1.9 \mu \mathrm{m}$ (see Methods Section E. for the setup and rod assembly protocol).

In low-ionic-strength aqueous suspensions, electrostatic repulsion [32] gives rise to a potential barrier which limits the formation of solid-solid interparticle contacts. A shallow secondary minimum then permits the formation of weak interparticle bonds, that can be broken using optical traps [33, 34]. In Supplementary Note 2 and Video we show that here, in contrast:

(a) For $I \geq 0.1 \mathrm{M}$, no repulsive barrier exists to limit contact formation

(b) Consistently, when two particles are approached, they form a contact that resists opening

Additionaly:

(c) Once formed, rods remain stable for hours when held by just two traps acting on the end particles.

Under the studied conditions, hence, when two particles are approched, they form solid-solid contacts that do not break by thermal activation over the timescale at which we observe macroscopic aging.

By analyzing rod images during the laser tweezer experiment (see Supplementary Methods), we access the bending force $f$ as a function of rod deflection $\delta$. Typical data, for a single rod, at different times after its formation, are reported in Fig. 3b. Such experiments, replicated at different ionic strengths between $0.10 \mathrm{M}$ and $0.20 \mathrm{M}$, yield the following observations:

(d) Under loading, rods bend according to the EulerBernoulli equation (see Supplementary Methods and Refs. [27, 28]), which demonstrates that contacts resist rolling.

(e) The bending modulus grows over time (Fig. 3b).

Point (d) suggests that the rod stiffness can be charac- terized by an effective single-bond rigidity,

$$
k_{0}=k_{\mathrm{b}} \times\left(\frac{L}{a}\right)^{3}
$$

with $L$ the rod length and $k_{b}$ the rod bending modulus deduced from the Euler-Bernouilli equation. Plotting $k_{0}$ vs $t$ for both 11 and 13 particles rods [Fig. 3c] confirms that $k_{0}$ is independent of $L$, and demonstrates that:

(f) flexural rigidity reaches a roughly logarithmic growth regime at later times.

Like $G^{\prime}(t), k_{0}(t)$ shows an upward curvature, so that the logarithmic scaling extends over less than a decade.

Besides, $k_{0}$ is $I$-independent at the considered salt concentrations: it expectedly results from the saturation, for $I \geq 0.1 \mathrm{M}$, of the zeta potential (at $\simeq-10 \mathrm{mV}$ ), hence of the charge density, of our particles (see [7] and Supplementary Note 2).

Two key conclusions may be drawn from the above observations. First, points $(\mathrm{a}-\mathrm{d})$ demonstrate the nonbarrier-limited formation of solid-solid, roll-resistant, contacts that do not break by thermal activation. This unambiguously supports that the rapid formation of contacts stabilizes suspension microstructures shortly after arrest. Second, the evolution of the effective flexural rigidity of rods demonstrates that solid-solid inter-particle contacts age.

\section{From contact to macroscopic aging}

This now brings us to question (II). For a fixed microstructure, we expect

$$
G^{\prime}(t)=\frac{S}{a} \times k_{0}(t)
$$

with $S$ a $t$-independent dimensionless quantity, characterizing the structure. This linear relation is indeed clearly supported by plotting $G^{\prime}(t)$ vs $k_{0}(t)$ for our $2 a \simeq 1.9 \mathrm{\mu m}$ particles (blue symbols in Fig. 4 a); it also applies to all the studied ionic strengths and packing fractions. These data show that, in the tested conditions, macroscopic aging results from the progressive stiffening of interparticle contacts, the microstructure being essentially frozen shortly after arrest.

Let us explore in more detail the possible origin of contact aging and its consequences in terms of macroscopic aging. We recall that interparticle solid-solid contacts are flat circular areas of radius

$$
a_{c}=A\left(\frac{3 \pi a^{2} W}{8 E^{*}}\right)^{1 / 3}
$$

where $A$ is a prefactor ranging from 1 to $3^{1 / 3} \simeq 1$.44 between the DMT and JKR limits [35-37]. For Stöber silica, as Young's modulus $E \simeq 30 \mathrm{GPa}$ and Poisson's ratio 

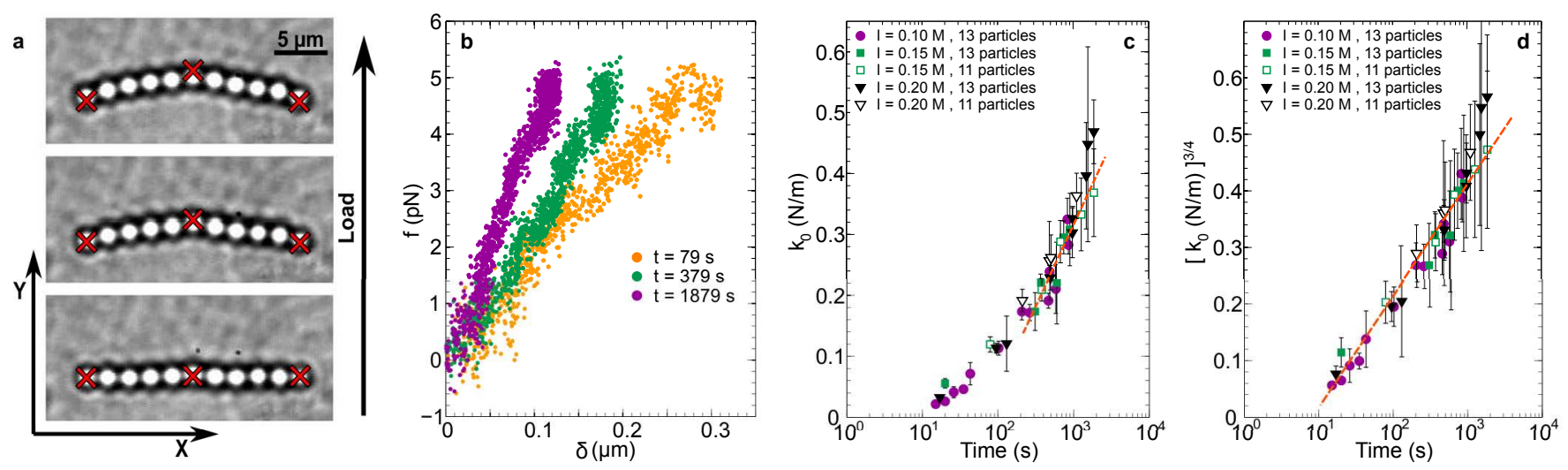

FIG. 3. Laser tweezer bending test. a: Three snapshots of an eleven-particle beam at different loading levels during the three-point bending test; red X's represent schematically the positions of optical traps; b: Force $f$ vs beam deflection $\delta$ after three aging times; c: Bond stiffness $k_{0}$ as a function of time $t$ for different ionic strengths, as measured from three-point bending tests with two rod lengths $L$ (error bars show the confidence interval of the linear fit of $f$ vs $\delta$ data); d: Log-lin plot of $\left[k_{0}\right]^{3 / 4}$ vs $t$ supporting that asymptotic growth scales as $\simeq[\log (t / \tau)]^{4 / 3}$ (see text for details).

$\nu \simeq 0.17$ [38], the reduced modulus $E^{*}=E /\left(1-\nu^{2}\right) / 2 \simeq$ $15 \mathrm{GPa}$. For a freshly formed contact, the surface energy $W \simeq 2 \gamma_{S L}$ with $\gamma_{S L}$ as the silica-water interfacial energy, which ranges between 50 and $100 \mathrm{~mJ} / \mathrm{m}^{2}$ [39]. The Tabor number $\lambda=\left(W^{2} a /\left(8\left(E^{*}\right)^{2} h^{3}\right)\right)^{1 / 3}$, with $h$ the gap (contact thickness), is small in the DMT limit, and large in the JKR one. It may be bounded above provided $W \lesssim 200 \mathrm{~mJ} / \mathrm{m}^{2}$, by taking $2 a \simeq 1.9 \mu \mathrm{m}$, an upper limit for the particle diameter, and $h \simeq 0.3 \mathrm{~nm}$, a lower value for the gap [40]. Therefore, $\lambda \lesssim 0.9$, which leads us to use the DMT estimate $A \simeq 1$ throughout. The ensuing analysis will support that contact stiffening results from a growth of $W$, yet, consistently, within $W \lesssim 200 \mathrm{~mJ} / \mathrm{m}^{2}$.

Since our rod bending data are consistent with the existence of a $L$-independent effective flexural modulus, we tentatively write $k_{0}$ as that of a beam of radius $a_{c}$ [27]:

$$
k_{0}=\frac{12 \pi E a_{c}^{4}}{a^{3}}=\frac{(3 \pi)^{7 / 3} E}{4 a^{1 / 3}}\left(\frac{W}{E^{*}}\right)^{4 / 3}
$$

Since $E$ and $E^{*}$ are bulk properties that do not change over time, this expression suggests that the growth of $k_{0}$ arises from that of $W$. There is, indeed, clear evidence in the literature that, at room temperature, the interface between two silica surfaces ages, in particular by the formation of siloxanes [41-45]. Yet, these works support that siloxane formation results in a logarithmic growth of the total surface energy $W$, which includes van der Walls and chemical contributions. When combined with expression (4), this idea suggests that $k_{0}^{3 / 4}$-not $k_{0}$ - grows logarithmically. Replotting our three-point bending data as $k_{0}^{3 / 4}$ vs $t$ [Fig. 3d], we do find the logarithmic scaling to be more evident than in Fig. 3c as it now extends over two decades in time.

This remarkable scaling supports that contact aging is akin to an increase over time of the total surface energy $W$ between the silica surfaces which causes $a_{c}$ and thus $k_{0}$ to grow. Using our $k_{0}$ measurements together with expression (4), we estimate $a_{c}$ to vary from 15 to $25 \mathrm{~nm}-$ small but not unreasonable values - while $W$ grows from 30 to $200 \mathrm{~mJ} / \mathrm{m}^{2}$ - a quite reasonable range [39].

Equation (4) predicts that the effective flexural rigidity depends on particle size only via the geometric prefactor $a^{-1 / 3}$. To test this scaling, we denote, from now on, $2 a^{\star}=1.9 \mu \mathrm{m}$ the diameter of the particles used in the three point bending test, and $k_{0}\left(a^{\star}\right)$ the corresponding bending modulus. Combining Eqs (2) and (4) yields,

$$
G^{\prime}=S \frac{\left(a^{\star}\right)^{1 / 3}}{a^{4 / 3}} k_{0}\left(a^{\star}, t\right)
$$

which predicts that, since $\left[k_{0}\left(a^{\star}\right)\right]^{3 / 4}$ does, $\left(G^{\prime}\right)^{3 / 4}$-not $G^{\prime}$ - grows logarithmically in time. We check this eventuality by plotting [Fig. 1b] $\left(G^{\prime}\right)^{3 / 4}$ vs $t$, and find a visible enhancement of the scaling range.

To further test Eq. (5), we report in Fig. 4a, $G^{\prime}(t)$ vs $k_{0}\left(a^{\star} ; t\right)$, for $a \neq a^{\star}$, and systematically find a linear relation $\left(G^{\prime} \simeq C+N k_{0}\left(a^{\star}\right)\right)$. The intercept $C$, occasionally non-zero, but always $<50 k P a$, appears to result from experimental errors. Indeed, (i) it is uncorrelated with our parameters $(a, I$, or $\phi)$; (ii) its values correspond to the indeterminacy of the origin of aging time in either rheometry or flexural bending experiments, and (iii) to errors of less that $0.03 \mathrm{~N} / \mathrm{m}$ on $k_{0}\left(a^{\star}\right)$, i.e. within our error bars. Using the fit parameters $C, N$, we reconstruct (brightly colored symbols in Fig. 1) $G^{\prime}(t)$ from the $k_{0}\left(a^{\star}\right)$ time series: the excellent collapse shows macroscopic modulus aging can be predicted using contact aging data for a single particle size.

Next, for each suspension, we use slope $N$ to estimate the structural parameter $S$ of equation (5):

$$
S \simeq \frac{a^{4 / 3}}{\left(a^{\star}\right)^{1 / 3}} N
$$



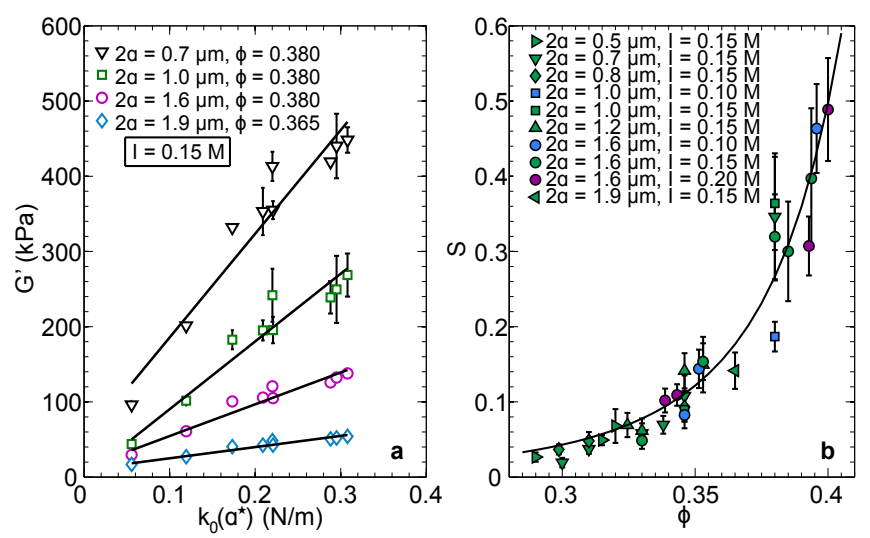

FIG. 4. Matching microscopic and macroscopic data. a: The macroscopic shear modulus $G^{\prime}(t)$ for four particle sizes vs the effective flexural rigidity $k_{0}\left(a^{\star} ; t\right)$ at the same aging time $t$, where $2 a^{\star}=1.9 \mu \mathrm{m}$; error bars show the standard deviation of $G^{\prime}$ data over centered 5 s intervals. b: Structural parameter $S$ as a function of packing fraction $\phi$ for different particle sizes (symbols) and ionic strengths (colors) and the curve $S=S_{0} /\left(\phi_{c}-\phi\right)^{\alpha}$ with $\phi_{c} \simeq 0.52$ and $\alpha=4$ (solid line); error bars show the confidence interval of the $G^{\prime}$ vs $k_{0}$ linear fit.

All these $S$ values, when plotted vs $\phi$ [Fig. 4b] remarkably collapse on a single curve. It confirms experimentally our model prediction for the particle size scaling of $G^{\prime}$, with the dimensionless prefactor $S$ depending neither on particle size, nor on ionic strength. Unsurprisingly, $S$ rapidly increases with $\phi$. The data suggest it diverges at a packing fraction $\phi_{c}$, since they match reasonably well the form $S=S_{0} /\left(\phi_{c}-\phi\right)^{\alpha}$, for example with $\phi_{c} \simeq 0.52$ and $\alpha=4$, as shown. Such an $S(\phi)$ relation, when combined with Eqs. (2) and (4), and with a logarithmic growth of $W$, provides a complete fit of our data set for varying $I, \phi, a$, and $t$.

Although these results are quite satisfactory, we remain surprised by, and cannot fully explain, the good agreement of our $k_{0}$ and $G^{\prime}$ data with the aging law $\propto[\log (t / \tau)]^{4 / 3}$, which emerges from our very simplified description of contacts. Thus, while we do bring compelling experimental evidence that contact aging drives macroscopic aging [Eq. (5)], we emphasize that theoretical progress on the precise mechanism of contact aging and on the link between contact rigidity and the modulus of particulate structures remains needed.

\section{A test of genericity}

The core result of our work is the experimental demonstration that macroscopic mechanical aging may arise solely from contact aging, with the microstructure being fixed. We expect that it should be a widespread
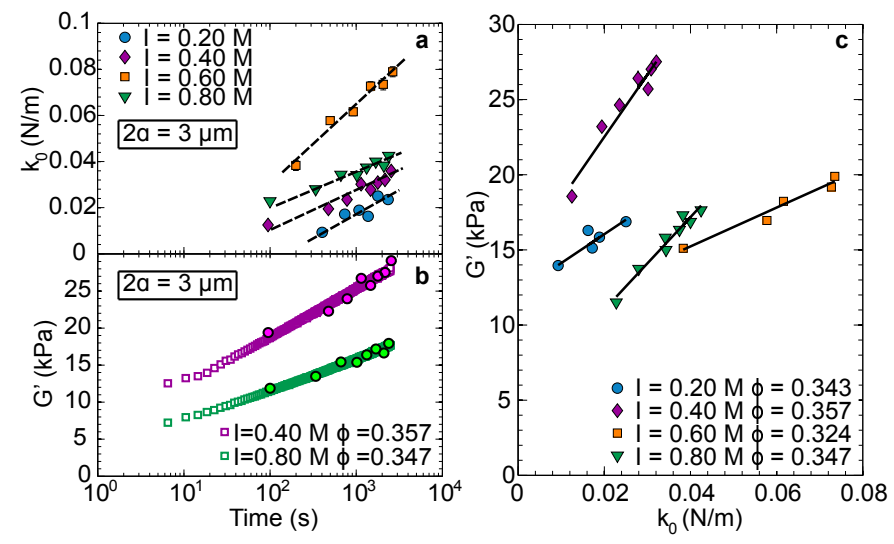

FIG. 5. Macroscopic and contact aging in PMMA suspensions. a: Time evolution of the bond stiffness for different salt concentrations $I$, measured with the three-point bending tests; b: Macroscopic shear modulus $G^{\prime}$ (colored squares) for two suspensions with $\phi \simeq 0.35$ and different ionic strengths along with the modulus reconstructed from the $k_{0}$ time series (brightly colored circles) using a linear fit as shown in the next panel; c: Shear modulus $G^{\prime}$ vs $k_{0}$ for the same aging times, showing that the two quantities are well described by a linear relationship.

phenomenon, since it is likely to apply as soon as the formation of interparticle contacts cannot be prevented.

To test this expectation, we have examined PMMA suspensions in $\mathrm{CaCl}_{2}$ salt solutions for a single particle size $2 a=3 \mu \mathrm{m}$ and $I$ values between 0.2 and $0.8 \mathrm{M}$. In all cases, we were again able to form stable rods (when held by their extremities). Moreover, in all studied cases, $k_{0}$ ages quasi-logarithmically (Fig. 5a).

These observations demonstrate the formation, between PMMA particles, of solid-solid contacts that age. Yet, in contrast with the case of silica particles, the bending stiffness [Fig. 5a] depends significantly and nonmonotonically on $I$. This feature was previously noted by Pantina and Furst [28], who attributed it to a particle charge reversal resulting from the affinity of $\mathrm{Ca}^{++}$cations with the PMMA surface. Noticeably, for PMMA particles, contact aging may be ascribed to the chain interdigitations occurring at the contact interface [32], hence via a quite different mechanism than in the case of silica.

Like $k_{0}$, the macroscopic shear modulus of PMMA suspensions [Fig. 5b], is quite sensitive to ionic strength. To test Eq. (2), we report in Fig. $5 \mathrm{c}$ our $G^{\prime}(t)$ vs $k_{0}(t)$ data for several ionic strengths. All of these plots are very clearly linear: $G^{\prime} \simeq C+N k_{0}$. However, in sharp contrast with the case of silica particle suspensions, the intercepts are large and cannot arise from experimental errors. It indicates that, at very early times, when the bending rigidity is negligibly small, PMMA suspensions already present an initial measurable macroscopic modulus $G_{0}^{\prime} \simeq C$ associated with the network of attractive interparticle bonds. The linearity of the $G^{\prime}$ vs $k_{0}$ plots supports that $G^{\prime}$ is just the sum of two contributions: 
$G_{0}^{\prime}$, which does not age, and $N k_{0}$, which does, due to contact aging. Indeed, the growth of $G^{\prime}(t)$ can be reconstructed from the $k_{0}(t)$ times series, using just the two fit parameters $G_{0}^{\prime}$ and $N$ : see the brightly colored symbols in Fig. 5b, which fall right atop the $G^{\prime}(t)$ curve. Our PMMA data thus demonstrate that, just as with silica suspensions, the growth of $G^{\prime}$ can be attributed to that of $k_{0}$, irrespectively of the value of the initial modulus $G_{0}^{\prime}$.

\section{Contact-driven aging}

In this paper, we have identified a single aging scenario, in two concentrated, ionic, model suspensions with particles of sharply different natures: first, the microstructure rapidly stabilizes due to the formation of irreversible contacts; then, macroscopic shear modulus aging proceeds due to contact stiffening, i.e. the growth of interparticle flexural rigidity.

It is noteworthy that our micron-sized silica particles should sediment over seconds if they were not interacting. Hence, the stability of our suspensions at moderate volume fractions (at least down to 33\% [7]) signals the rapid formation of attractive contacts. Besides, in both our systems, since rejuvenation is easily achieved by preshearing, solid-solid contacts, although stable under thermal fluctuations, break under moderate stresses. These observations underline that suspensions may be both homogeneous and easily reshuffled by shearing, even when particles form solid-solid contacts that are stable at rest. Stability and homogeneity hence may be facilitated by solid-solid contact formation.

The key condition for the relevance of our scenario is that the formation of solid-solid contacts is essentially not barrier limited, i.e. significantly faster than other processes (diffusion, sedimentation). Indeed, typically, solid-solid contacts between micron-sized colloidal particles involve adhesion energies in the thousand $k T$ [32] range, hence cannot be opened by thermal activation at room temperature $[33,34]$. So, at moderate or high packing fractions, such suspensions rapidly freeze into thermally irreversible structures. Additionally: (i) solidsolid contacts generically display roll-resistance [46], due to surface roughness; (ii) contacts age under quite broad conditions, although via different routes depending on the physico-chemistries of the particles and suspending fluid.
For these reasons, our scenario-rapid stabilization by contact formation followed by contact-driven mechanical aging - is expected to arise as soon as the ionic concentration is sufficient to screen out Coulombic repulsion. This is likely the case in many materials of practical importance, such as cements at a young age [25], sediments, ceramics, sewage sludges, mine tailings, etc, which present ionic concentrations typically larger than those considered here. Whenever our scenario applies, the quantitative relations we have identified between contact and mechanical aging provide a plausible starting point for predictive models.

The precise domain of relevance of our scenario, of course, will need to be assessed case-by-case. Contact aging may also compete with structural aging; for example, if the formation of contacts in not as rapid as herei.e. is limited by non-vanishing but small barriers - or if solid-solid contacts are weak enough to open by thermal activation. Progress on these questions will open the route towards the possibility to tune mechanical aging by controlling and tailoring the surface chemistry of particles, a fascinating perspective in many applications.

Acknowledgements. This work has benefited from a French government Grant managed by ANR within the framework of the National Program Investments for the Future ANR-11-LABX-0022-01. FB's stay at the University of Delaware was supported by University Paris-Est.

Author Contributions. XC, JG, and AL conceived and supervised the project. JG and JF designed the macroscopic shear modulus aging protocol and obtained data on silica suspensions. JG supervised sample preparation and all experiments. EF designed the laser tweezer three-point flexural test and supervised its use. FB obtained complementary macroscopic shear modulus data, designed the two-compartment cell, performed flexural aging measurements with EF and JG. All project participants contributed to the interpretation of experimental data, model construction, and article planning and writing.

Author Information. Reprints and permissions information is available at www.nature.com/reprints. Correspondence and requests for materials should be addressed to $\mathrm{XC}$ and $\mathrm{AL}$.

Competing interests. The authors declare no competing interests.
[1] Abou, B., Bonn, D. \& Meunier, J. Aging dynamics in a colloidal glass. Phys. Rev. E 64, 021510 (2001). URL https://link.aps.org/doi/10.1103/ PhysRevE.64.021510.

[2] Derec, C., Ducouret, G., Ajdari, A. \& Lequeux, F. Aging and nonlinear rheology in suspensions of polyethylene oxide-protected silica particles. Phys. Rev. E 67,
061403 (2003). URL https://link.aps.org/doi/10. 1103/PhysRevE.67.061403.

[3] Coussot, P., Tabuteau, H., Chateau, X., Tocquer, L. \& Ovarlez, G. Aging and solid or liquid behavior in pastes. Journal of Rheology 50, 975-994 (2006). URL https:// doi.org/10.1122/1.2337259.

[4] Ovarlez, G. \& Coussot, P. Physical age of soft-jammed 
systems. Phys. Rev. E 76, 011406 (2007). URL https:// link.aps.org/doi/10.1103/PhysRevE.76.011406.

[5] Ovarlez, G. \& Chateau, X. Influence of shear stress applied during flow stoppage and rest period on the mechanical properties of thixotropic suspensions. Phys. Rev. E 77, 061403 (2008). URL https://link.aps.org/doi/ 10.1103/PhysRevE.77.061403.

[6] Guo, H., Ramakrishnan, S., Harden, J. L. \& Leheny, R. L. Gel formation and aging in weakly attractive nanocolloid suspensions at intermediate concentrations. The Journal of Chemical Physics 135, 154903 (2011). URL https://doi.org/10.1063/1.3653380.

[7] Fusier, J., Goyon, J., Chateau, X. \& Toussaint, F. Rheology signature of flocculated silica suspensions. Journal of Rheology 62, 753-771 (2018). URL https://doi.org/ 10.1122/1.5031897.

[8] Dinsmore, A. D., Weeks, E. R., Prasad, V., Levitt, A. C. \& Weitz, D. A. Three-dimensional confocal microscopy of colloids. Appl. Opt. 40, 4152-4159 (2001). URL http:// ao. osa. org/abstract . cfm?URI=ao-40-24-4152.

[9] Dinsmore, A. D. \& Weitz, D. A. Direct imaging of threedimensional structure and topology of colloidal gels. Journal of Physics-Condensed Matter 14, 7581-7597 (2002). URL https://doi.org/10.1088\%2F0953-8984 $\% 2$ F14\%2F33\%2F303. Times Cited: 82 .

[10] Prasad, V., Semwogerere, D. \& Weeks, E. R. Confocal microscopy of colloids. Journal of Physics: Condensed Matter 19, 113102 (2007). URL http://stacks.iop. org/0953-8984/19/i=11/a=113102.

[11] Dibble, C. J., Kogan, M. \& Solomon, M. J. Structure and dynamics of colloidal depletion gels: Coincidence of transitions and heterogeneity. Phys. Rev. E 74, 041403 (2006). URL https://link.aps.org/doi/ 10.1103/PhysRevE.74.041403.

[12] Whitaker, K. A. et al. Colloidal gel elasticity arises from the packing of locally glassy clusters. Nature Communications 10, 2237 (2019). URL https://doi.org/10. 1038/s41467-019-10039-w.

[13] Cipelletti, L., Manley, S., Ball, R. C. \& Weitz, D. A. Universal aging features in the restructuring of fractal colloidal gels. Phys. Rev. Lett. 84, 2275-2278 (2000). URL https://link.aps.org/doi/10.1103/ PhysRevLett.84.2275.

[14] Bissig, H., Romer, S., Cipelletti, L., Trappe, V. \& Schurtenberger, P. Intermittent dynamics and hyperaging in dense colloidal gels. PhysChemComm 6, 21-23 (2003). URL http://dx.doi.org/10.1039/B211806H.

[15] Cipelletti, L. et al. Universal non-diffusive slow dynamics in aging soft matter. Faraday Discuss. 123, 237-251 (2003). URL http://dx.doi.org/10.1039/B204495A.

[16] Barnes, H. A. Thixotropya review. Journal of Non-Newtonian Fluid Mechanics 70, $1-33$ (1997). URL http://www. sciencedirect.com/science/ article/pii/S0377025797000049.

[17] Cipelletti, L. \& Ramos, L. Slow dynamics in glassy soft matter. Journal of Physics: Condensed Matter 17, R253 (2005). URL http://stacks.iop.org/0953-8984/17/ $i=6 / a=R 01$.

[18] Mewis, J. \& Wagner, N. J. Colloidal Suspension Rheology. Cambridge Series in Chemical Engineering (Cambridge University Press, 2011). URL https://www.cambridge.org/ core/books/colloidal-suspension-rheology/ E4C1D16944B043534881158BC62D3E59.
[19] Bouzid, M., Colombo, J., Barbosa, L. V. \& Del Gado, E. Elastically driven intermittent microscopic dynamics in soft solids. Nature Communications 8, 15846 (2017). URL https://doi.org/10.1038/ncomms15846.

[20] Dieterich, J. H. \& Kilgore, B. D. Imaging surface contacts: power law contact distributions and contact stresses in quartz, calcite, glass and acrylic plastic. Tectonophysics 256, 219 - 239 (1996). URL http://www. sciencedirect.com/science/ article/pii/0040195195001654.

[21] Persson, B. N. J. Sliding Friction: Physical Principles and Applications (Nanoscience and Technology) (Springer Verlag, 2000).

[22] Baumberger, T. \& Caroli, C. Solid friction from stickslip down to pinning and aging. Advances In Physics 55, 279-348 (2006).

[23] Vandamme, M. \& Ulm, F.-J. Nanogranular origin of concrete creep. Proceedings of the National Academy of Sciences 106, 10552-10557 (2009). URL https://www.pnas.org/content/106/26/10552. https://www.pnas.org/content/106/26/10552.full.pdf.

[24] Ioannidou, K. et al. The crucial effect of early-stage gelation on the mechanical properties of cement hydrates. Nature Communications 7, 12106 (2016). URL https:// doi.org/10.1038/ncomms12106.

[25] Roussel, N., Ovarlez, G., Garrault, S. \& Brumaud, C. The origins of thixotropy of fresh cement pastes. Cement and Concrete Research 42, 148 - 157 (2012). URL http://www. sciencedirect.com/science/ article/pii/S0008884611002353.

[26] Manley, S. et al. Time-dependent strength of colloidal gels. Phys. Rev. Lett. 95, 048302 (2005). URL https:// link.aps.org/doi/10.1103/PhysRevLett.95.048302.

[27] Pantina, J. P. \& Furst, E. M. Elasticity and critical bending moment of model colloidal aggregates. Phys. Rev. Lett. 94, 138301 (2005). URL https://link.aps. org/doi/10.1103/PhysRevLett.94.138301.

[28] Pantina, J. P. \& Furst, E. M. Colloidal aggregate micromechanics in the presence of divalent ions. Langmuir 22, 5282-5288 (2006). URL https://doi.org/10.1021/ la0534120. PMID: 16732653.

[29] Meng, B., Wu, J., Li, Y. \& Lou, L. Aging process of the bond between colloidal particles measured using laser tweezers. Colloids and Surfaces A: Physicochemical and Engineering Aspects 322, 253 - 255 (2008). URL http://www. sciencedirect.com/science/ article/pii/S092777570800126X.

[30] Buscall, R., Mills, P. D. A., Goodwin, J. W. \& Lawson, D. W. Scaling behaviour of the rheology of aggregate networks formed from colloidal particles. J. Chem. Soc., Faraday Trans. 1 84, 4249-4260 (1988). URL http:// dx.doi.org/10.1039/F19888404249.

[31] Russel, W. B., Saville, D. A. \& Schowalter, W. R. Colloidal Dispersions. Cambridge Monographs on Mechanics (Cambridge University Press, 1989). URL https://www . cambridge.org/core/books/colloidal-dispersions/ A880F349E6ECA53C2E65D0FDEDABB091.

[32] Israelachvili, J. N. 17 - adhesion and wetting phenomena. In Israelachvili, J. N. (ed.) Intermolecular and Surface Forces (Third Edition), 415 - 467 (Academic Press, San Diego, 2011), third edition edn. URL http://www.sciencedirect.com/science/ article/pii/B978012375182910017X.

[33] Swan, J. W., Shindel, M. M. \& Furst, E. M. Mea- 
suring thermal rupture force distributions from an ensemble of trajectories. Phys. Rev. Lett. 109, 198302 (2012). URL https://link.aps.org/doi/10.1103/ PhysRevLett.109.198302.

[34] Whitaker, K. A. \& Furst, E. M. Bond rupture between colloidal particles with a depletion interaction. Journal of Rheology 60, 517-529 (2016). URL https://doi .org/ 10.1122/1.4947043. https://doi.org/10.1122/1.4947043.

[35] Derjaguin, B., Muller, V. \& Toporov, Y. Effect of contact deformations on the adhesion of particles. Journal of Colloid and Interface Science 53, 314 - 326 (1975). URL http://www. sciencedirect.com/science/ article/pii/0021979775900181.

[36] Johnson, K. L., Kendall, K. \& Roberts, A. D. Surface energy and the contact of elastic solids. Proceedings of the Royal Society of London A: Mathematical, Physical and Engineering Sciences 324, 301-313 (1971). URL http://rspa.royalsocietypublishing. org/content/324/1558/301.

[37] Tabor, D. Surface forces and surface interactions. Journal of Colloid and Interface Science 58, 2 - 13 (1977). URL http://www. sciencedirect.com/science/ article/pii/0021979777903666. International Conference on Colloids and Surfaces.

[38] Paul, J., Romeis, S., Tomas, J. \& Peukert, W. A review of models for single particle compression and their application to silica microspheres. Advanced Powder Technology 25, 136 - 153 (2014). URL http://www. sciencedirect. com/science/article/pii/S0921883113001891.

[39] Iler, K. The Chemistry of Silica: Solubility, Polymerization, Colloid and Surface Properties and Biochemistry of Silica (John Wiley and Sons Inc, 1979). URL https:// www . wiley.com/en-us/The+Chemistry+of+Silica $\% 3 \mathrm{~A}+$ Solubility $\% 2 \mathrm{C}+$ Polymerization $\% 2 \mathrm{C}+\mathrm{Colloid}+$ and+Surface+Properties+and+Biochemistry+

[40] Guleryuz, H., Røyset, A. K., Kaus, I., Filiàtre, C. \& Einarsrud, M.-A. Afm measurements of forces between silica surfaces. Journal of Sol-Gel Science and Technology 62, 460-469 (2012). URL https://doi.org/10.1007/ s10971-012-2750-6.

[41] Vigil, G., Xu, Z., Steinberg, S. \& Israelachvili, J. Interactions of silica surfaces. Journal of Colloid and Interface Science 165, 367 - 385 (1994). URL http://www. sciencedirect.com/science/ article/pii/S0021979784712422.

[42] Li, Q., Tullis, T. E., Goldsby, D. \& Carpick, R. W. Frictional ageing from interfacial bonding and the origins of rate and state friction. Nature 480, $233 \mathrm{EP}-(2011)$. URL https://doi.org/10.1038/nature10589.

[43] Liu, Y. \& Szlufarska, I. Chemical origins of frictional aging. Phys. Rev. Lett. 109, 186102 (2012). URL https:// link.aps.org/doi/10.1103/PhysRevLett.109.186102.

[44] Tian, K. et al. Load and time dependence of interfacial chemical bond-induced friction at the nanoscale. Phys. Rev. Lett. 118, 076103 (2017). URL https://link.aps. org/doi/10.1103/PhysRevLett.118.076103.

[45] Li, Z., Pastewka, L. \& Szlufarska, I. Chemical aging of large-scale randomly rough frictional contacts. Phys. Rev. E 98, 023001 (2018). URL https://link .aps.org/ doi/10.1103/PhysRevE.98.023001.

[46] Del Gado, E. \& Kob, W. A microscopic model for colloidal gels with directional effective interactions: network induced glassy dynamics. Soft Matter 6, 1547-1558 (2010). URL http://dx.doi.org/10.1039/B916813C.

\section{METHODS}

\section{Particles}

Silica micro particles are produced by a single growth step Stöber synthesis [47] in a semibatch process (continuous addition). This is considered to be the best method to ensure sphericity and monodispersity [48]. We add small amounts of electrolyte ( $\mathrm{NaCl}$, Sigma-Aldrich) to the initial ammonia solution, at concentrations ranging from 1 to $4 \mathrm{mM}$, to achieve better control of the final particle size [49]. Particles are separated by centrifugation and then washed several times with ethanol 96\% (GPR Rectapur VWR) and with de-ionized water (Aquadem $18 \mathrm{M} \Omega$ ) to eliminate impurities and solvents. They are finally dried at $60^{\circ} \mathrm{C}$ for several days.

The particle sizes in all batches (for both rheometry and bending test) were measured by DLS (Malvern Zetasizer Nano S). In addition, we collected SEM micrographs (Zeiss Neon 40 EsB FIB-SEM) of the particles used in Laser Tweezers experiments to have more accurate size measurements; we found them to present a mean diameter $d=(1860 \pm 90) \mathrm{nm}$ and $5 \%$ polydispersity, which compares well with the DLS results $(d=(1960 \pm 430) n m)$.

PMMA particles were purchased from MicroBeads (Spheromers CA3). Particle sizes are distributed around $3 \mu \mathrm{m}$ with a $15 \%$ polydispersity. The particles were washed several times with ethanol and de-ionized water +
betSile usca-p-9780471024040.

\section{Suspensions}

Suspensions are prepared following the protocol shown by Fusier et al. [7] to permit experimental reproducibility. A small amount of dry silica powder (of known weight) is dispersed and left for several hours in a large volume of de-ionized water; the mixture is then centrifugated at $5000 \mathrm{rpm}$ for $5 \mathrm{mn}$ to eliminate the gross part of water; salt is dissolved in the volume of water needed to reach the target packing fraction $\phi$ and ionic strength $I$; finally, the centrifugated particles and saline water are vigorously mixed until a homogeneous suspension is obtained.

A slightly different procedure is required for PMMA suspensions, as the hydrophobicity of the PMMA surface [50] complicates the dispersion process. Dispersed PMMA particles are stirred for 2 days and then allowed to sediment overnight; most of the excess water along with some hydrophobic particles adsorbed at the airliquid interface have to be removed with a syringe; centrifugation is performed at $5500 \mathrm{rpm}$ for 20 minutes. 


\section{Rheometry}

Rheology is performed using a stress-controlled (Malvern Kinexus Ultra +) rheometer, in a thin $(1.25 \mathrm{~mm})$ gap Couette geometry between roughned cylinders to avoid slippage. After the rheometer cup is filled, sonification is performed for 2 minutes to remove bubbles.

Prior to each measurement, the system is presheared at $200 \mathrm{~s}^{-1}$ for $3 \mathrm{~min}$, a high strain-rate corresponding to two to three times the yield stress [7]. This brings our suspensions into a well-defined initial state, and insures the reproducibility of the ensuing shear modulus evolution [7]. The end of pre-shear is taken as the origin of aging time.

The shear modulus is then measured at different times by applying an oscillatory shear stress at $1 \mathrm{~Hz}$ (to avoid retro-action noise), using a feedback loop to achieve a strain amplitude of $10^{-4}$, and thus stay within the linear regime [7].

\section{Confocal microscopy}

We use a confocal microscope (Zeiss LSM 700) with a $100 \times$ NA 1.4 oil immersion objective (Zeiss PlanAPOCHROMAT). We image silica particles in a $60 / 40$ wt\% mixture of water/glycerol with a small amount of fluorescein. Our confocal microscopy measurements are performed in a single focal plane, at depth $\approx 15 \mu \mathrm{m}$. The acquisition time of an image $(0.5 \mathrm{~s})$ is quite short compared with our observation timescale. Particle reconstruction is performed using a standard centroid-based algorithm [51]. It usually permits to position particles within a tenth of a pixel, which corresponds to $\simeq 12 \mathrm{~nm}$ in our $2 \mathrm{D}$ snapshots $[51,52]$. However, due to the residual index mismatch our resolution is slightly lower.

\section{Bending test}

We use the custom-made tweezers setup of Ref. [53] which permits to manipulate multiple particles by timesharing a single laser beam [see details in the referenced paper]. Prior to each experiment, the trap stiffness, $k_{\text {trap }}$, is measured by examining the thermal fluctuations of a trapped bead, the position of which is resolved with nanometric accuracy by back-focal plane interferometry [54]. The sample cells are made using a double-sided adhesive spacer to create a gap between a microscope slide (Fisher, size $25 \times 75 \times 1 \mathrm{~mm}^{3}$ ) and a cover-slip (Fisher, size $\left.22 \times 30 \times 0.17 \mathrm{~mm}^{3}\right)$.

We have studied beams formed using a single particle size $2 a=1.9 \mu \mathrm{m}$. This choice results from a compromise between two constraints: 1 ) the higher $a$, the broader the range of accessible forces (set by the silica/water optical index contrast [55]) and the better the evaluation of particle positions at sub-pixel accuracy; 2) it is difficult to synthesize large monodisperse particles. The chosen $a$ value limits polydispersity to $5 \%$, while allowing us to access the desired rod properties.

A difficulty we encountered is that, at the targeted salt concentrations, both $\mathrm{SiO}_{2}$ and PMMA particles, which sediment due to density contrast, stick so strongly to the coverslip that they cannot be detached with the tweezers. To be able to manipulate these particles, we need to postpone the time when particles enter the saline solution. We achieve this by dividing the cell into two compartments carved in the adhesive spacer: a small $(7 \mu \mathrm{L})$ one, where a highly diluted $\left(\phi \approx 10^{-4}\right)$ suspension of particles in pure water is introduced; a large one $(50 \mu \mathrm{L})$, where the solution is introduced. To prevent drying, the whole cell is sealed using a fast UV-curing epoxy (Norland Products, NOA 81) immediately after the introduction of the ionic solution and suspended particles. The two compartments are in open contact with each other through a small aperture, which sufficiently slows down salt diffusion that particles, although they sediment, do not stick to the coverslip, and can be handled, for nearly 2 hours. The size difference between the two compartments guarantees that even after the complete diffusive dispersion of salt the relative error in the ionic strength remains smaller than $\approx 5 \%$.

We use beams of 11 or 13 particles. They are assembled using an array of the same number of time-shared traps separated by a distance $(\approx 2.5 \mu \mathrm{m})$ larger than the particle diameter. The array is filled with particles inside the small compartment and then moved through the aperture to the ionic solution (large compartment) by means of the motorized microscope stage. The beam is finally formed by reducing the separation between the traps until vdW forces induce aggregation [27].

The full aggregation of a beam takes a time of order a minute due to fluctuations caused primarily by Brownian motion. The formation of the last contact is taken as the origin of time for the beam evolution. Thereafter, the beam is held only by three traps: two at its extremities, one at its center. Bending is enforced by translating the center trap perpendicularly to the chain, at a velocity slow enough to create negligible hydrodynamic drag (30 $\mathrm{nm} / \mathrm{s}$ ), while the two other traps are held fixed. We call $x$ the axis passing through the end particles of the rod, and $y$ the perpendicular axis in the focal plane of the microscope (see Fig. 3a). Microscope images, having a pixel size $0.117 \mu \mathrm{m}$, provide particle positions at a subpixel $(1 / 10 \mathrm{th})$ precision $\approx 10 \mathrm{~nm}[51]$.

The trap stiffnesses being known, the bending force $f$ is obtained from the $y$-displacements of the end particles away from their pre-loading positions, which correspond to the location of the (fixed) traps; the deflection is estimated as the differences in $y$ of the end and central particles. The raw data thus inferred from $y$ displacements alone, however, have to be corrected to take into account mainly two problems: (a) rods are not strictly linear and 
(b) the optical traps and microscope focal planes are not perfectly aligned. In Supplementary Methods, we explain how we do so and improve our measurements of $f$ and $\delta$.

Data availability. Figure source data are provided online; other data used in this work are available from the authors.

Code availability. The software used in this work is available from the authors.
[47] Stober, W., Fink, A. \& Bohn, E. Controlled growth of monodisperse silica spheres in the micron size range. Journal of Colloid and Interface Science 26, $62-69$ (1968). URL http://www.sciencedirect.com/science/ article/pii/0021979768902725.

[48] Do, K. K. \& Taik, K. H. New process for the preparation of monodispersed, spherical silica particles. Journal of the American Ceramic Society 85, 1107-1113 (2002). URL https://onlinelibrary.wiley.com/doi/abs/10. 1111/j.1151-2916.2002.tb00230.x.

[49] Nakabayashi, H. et al. Electrolyte-added one-pot synthesis for producing monodisperse, micrometer-sized silica particles up to $7 \mu \mathrm{m}$. Langmuir 26, 7512-7515 (2010). URL https://doi.org/10.1021/la904316f.

[50] Laxton, P. B. \& Berg, J. C. Investigation of the link between micromechanical interparticle bond rigidity measurements and macroscopic shear moduli of colloidal gels. Colloids and Surfaces A: Physicochemical and Engineering Aspects 301, 137 - 140 (2007). URL http://www. sciencedirect.com/science/ article/pii/S0927775706009708.

[51] Crocker, J. C. \& Grier, D. G. Methods of dig- ital video microscopy for colloidal studies. Journal of Colloid and Interface Science 179, 298 - 310 (1996). URL http://www.sciencedirect.com/science/ article/pii/S0021979796902179.

[52] Gao, Y. X. \& Kilfoil, M. L. Accurate detection and complete tracking of large populations of features in three dimensions. Optics Express 17, 4685-4704 (2009).

[53] Pantina, J. P. \& Furst, E. M. Directed assembly and rupture mechanics of colloidal aggregates. Langmuir 20, 3940-3946 (2004). URL https://doi.org/10.1021/ la0364338. PMID: 15969383.

[54] Shindel, M. M., Swan, J. W. \& Furst, E. M. Calibration of an optical tweezer microrheometer by sequential impulse response. Rheologica Acta 52, 455-465 (2013).

[55] Nieminen, T. A., Knner, G., Heckenberg, N. R. \& RubinszteinDunlop, H. Physics of optical tweezers. In Laser Manipulation of Cells and Tissues, vol. 82 of Methods in Cell Biology, 207 - 236 (Academic Press, 2007). URL http://www.sciencedirect.com/science/ article/pii/S0091679X06820066. 